\title{
Avulsion fracture of the calcaneus
}

\author{
Ross Berringer MD
}

- Cite as: CMAJ 2018 November 12;190:E1337. doi: 10.1503/cmaj.180248

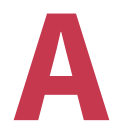

75-year-old woman was ascending an icy staircase when she had a sudden onset of right calf and heel pain, which resulted in abrupt dorsiflexion of her plantar-flexed right foot. She presented to the emergency department. On initial examination, she had a palpable soft tissue deficit immediately superior to the calcaneus. A Thompson test was positive, and the initial diagnosis was a rupture of the Achilles tendon. Given the patient's age, immobilization in plantar flexion and elective orthopedic referral were proposed as a treatment plan. When the patient was reassessed by the staff physician, the presence of a gap was confirmed. However, a sharp bony ridge was also noted, palpable about $4 \mathrm{~cm}$ above the calcaneus in addition to the previously noted gap. Skin tenting was present. There was no apparent bruising. Radiographs of the foot showed a type II avulsion fracture of the posterior superior calcaneus (Figure 1). The orthopedic service was consulted and the patient underwent urgent surgical fixation of the avulsed fragment (Figure 2). The patient made an uneventful recovery.

Less than $3 \%{ }^{1}$ of calcaneal fractures involve the tuberosity. As with rupture of the Achilles tendon, the mechanisms of injury are forced plantar flexion or abrupt dorsiflexion. Four types of calcaneal fractures are generally described. ${ }^{2}$ Type I fractures are most common and are characterized by small avulsions of the calcaneal tuberosity. Type II fractures are also avulsion injuries, but the bone fragment is larger and often "beaklike." Skin tenting may be present, and closed reduction is indicated if repair will be substantially delayed. Older women are at high risk for this type of injury. Type III and IV fractures are also avulsion injuries with varying degrees of damage to the Achilles tendon. Treatment is usually surgical, although some type III and IV injuries may be treated conservatively. ${ }^{1}$ Careful clinical examination should alert the clinician to the possibility of this type of calcaneal fracture, and allow differentiation between this injury and the more common Achilles tendon rupture.

If the bony fragment had not been palpated, this patient would have been treated nonoperatively with a presumed rupture of the Achilles tendon, which would likely have led to a poor clinical outcome. Plain radiography and computed tomography are adequate for bony detail, but ultrasonography and magnetic resonance imaging may be necessary for associated Achilles tendon injury. ${ }^{1,2}$

\section{References}

1. Rijal L, Sagar C, Adhikari D, et al. Calcaneal tuberosity avulsion fracture: an unusual variant. J Foot Ankle Surg 2012;51:666-8.

2. Lee SM, Huh SW, Chung JW, et al. Avulsion fracture of the calcaneal tuberosity: classification and its characteristics. Clin Orthop Surg 2012;4:134-8.

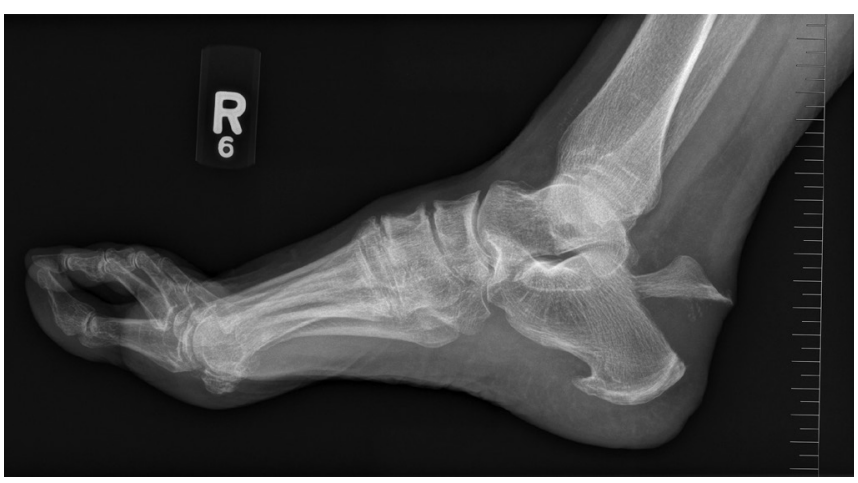

Figure 1: Radiograph of the right foot showing a type II avulsion fracture of the posterior superior calcaneus in a 75-year-old woman.

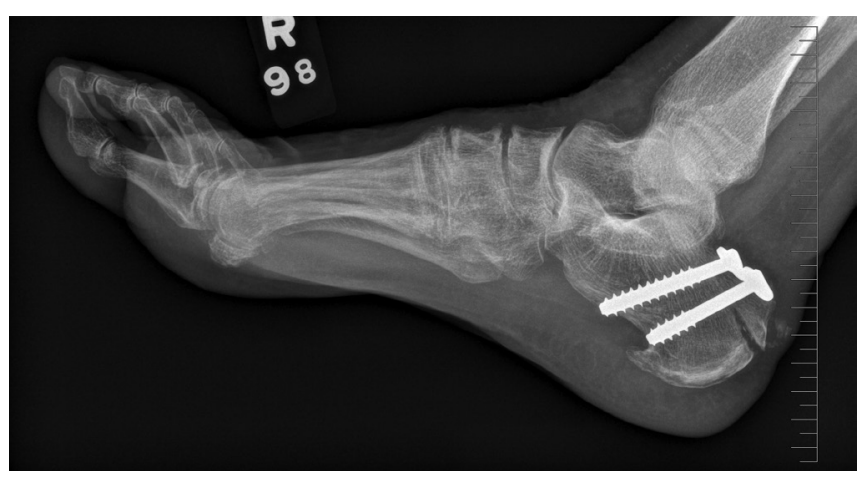

Figure 2: Postoperative radiograph.

Competing interests: None declared.

This article has been peer reviewed.

The author has obtained patient consent.

Affiliation: Providence Health Care, Vancouver, BC

Correspondence to: Ross Berringer, ross.berringer@gmail.com

Clinical images are chosen because they are particularly intriguing, classic or dramatic. Submissions of clear, appropriately labelled highresolution images must be accompanied by a figure caption. A brief explanation (300 words maximum) of the educational importance of the images with minimal references is required. The patient's written consent for publication must be obtained before submission. 